\title{
Introduction to MODELICA
}

\author{
P. Moreaux \\ LISTIC - Polytech Annecy-Chambéry \\ Université de Savoie - France \\ April 10th, 2012
}




\section{Introduction to MODELICA}

\section{P. Moreaux}

LISTIC - Polytech Annecy-Chambéry

Université de Savoie - France

April 10th, 2012

\section{SIMUREX 2012}

Cargèse, France - April 10-14, 2012 


\section{Overview}

- Modelica - where to get Information

- Main features

- Building Modelica models

- Some programming details

- Structuring - reuse 


\section{Overview}

- Modelica - where to get Information

- Main features

- Building Modelica models

- Some programming details

- Structuring - reuse 


\section{More information...}

\section{MODE L I C A}

- Modelica Association: www.modelica.org

- OpenModelica: www.openmodelica.org

" "The" book:

- Peter Fritzson,

Principles of Object Oriented

Modeling and Simulation

with Modelica 2.1,

Wiley-IEEE Press, 2004

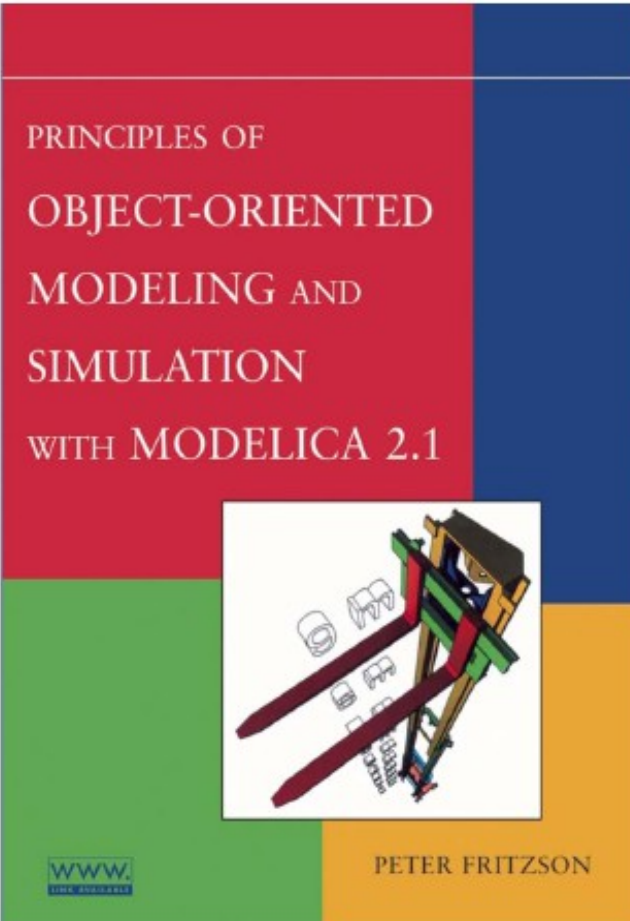




\section{Credits for this tutorial - Thanks to ...}

- A lot of very good, detailed, topic specific tutorials are available, see: http://modelica.org/

- Among them:

- Peter Fritzson, Introduction to Object-Oriented Modeling and Simulation with OpenModelica, (2006)

- Mohsen Torabzadeh-Tari (Linköping University), Introduction to Object-Oriented Modeling, Simulation and Control with Modelica, (May 2011).

- Martin Otter (DLR-RM and Chairman of Modelica Association), Modelica Overview, 2009

- Sébastien FURIC, R\&D Engineer, LMS (INSA Lyon, 2007) 


\section{Modelica - status}

- Free, open language

- Specification available at: www.modelica.org

- Developed since 1997 (1.0), V 3.2 released March 2010

- Modelica Association established 2000 in Linköping Open, non-profit organization Industry and Academia 


\section{Overview}

- Modelica - where to get Information

- Main features

- Building Modelica models

- Some programming details

- Structuring - reuse 


\section{Modelica - main goals}

- Modeling the dynamic behavior of technical systems consisting of components from, e.g., mechanical, electrical, thermal, hydraulic, pneumatic, fluid, control and other domains in a convenient way.

- Models are described by differential, algebraic, and discrete equations.

- No description by partial differential equations, i.e., no FEM (finite element method) and no CFD (computational fluid dynamics), but using results of, e.g., FEM programs. 


\section{Modelica - main features (1)}

- Declarative language (acausal)

- Equations and mathematical functions allow acausal modelling (high level specification, increased correctness)

- A single modelling framework

- Multi-domain modeling

- electrical, mechanical, thermodynamic, hydraulic, biological, control, event, real-time, etc... domains

- Hybrid modeling: continuous-time + discrete-time

- Modular programming! Components; connections

- Hierarchical system architecture capabilities 


\section{Modelica - main features (2)}

- Several basic constructs (model, block, record, ...) but technically,

- Everything is a class

(warning : not a OO language!)

- Strongly typed object-oriented language with a general class concept, Java \& MATLAB-like syntax

- Efficient, non-proprietary

- Efficiency comparable to C; advanced equation compilation,

- e.g. 300000 equations, 150 000 lines on standard PC

- Several environments for Visual component programming

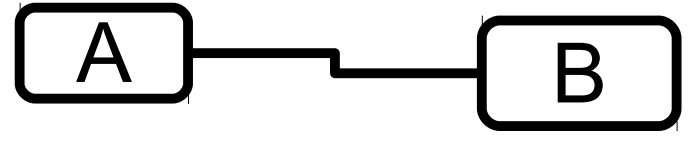




\section{Acausal versus causal modeling}

- Acausal model (Modelica)
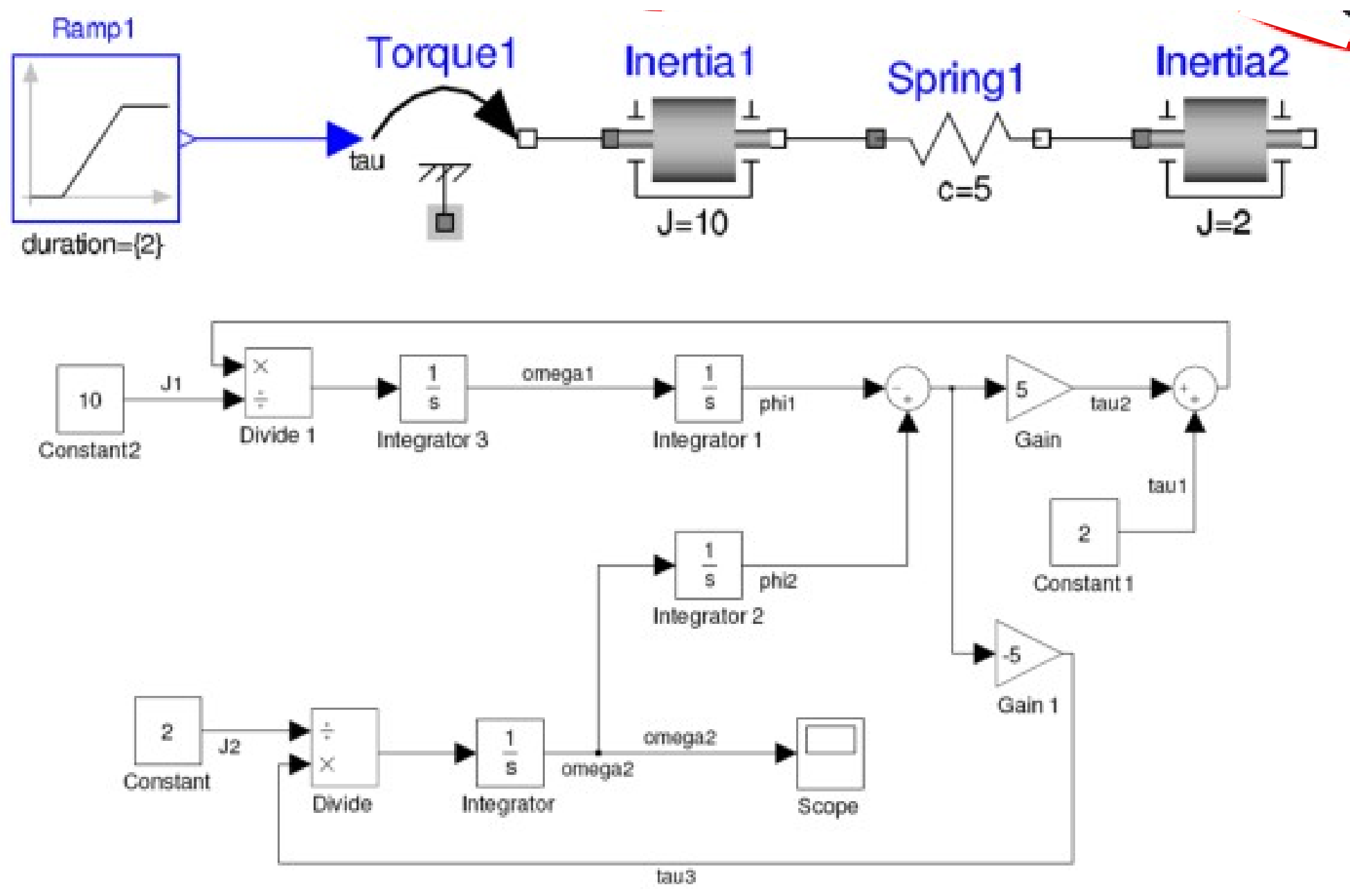

- Causal block-based model (Simulink) 


\section{Modelica vs Simulink Block Oriented Modeling

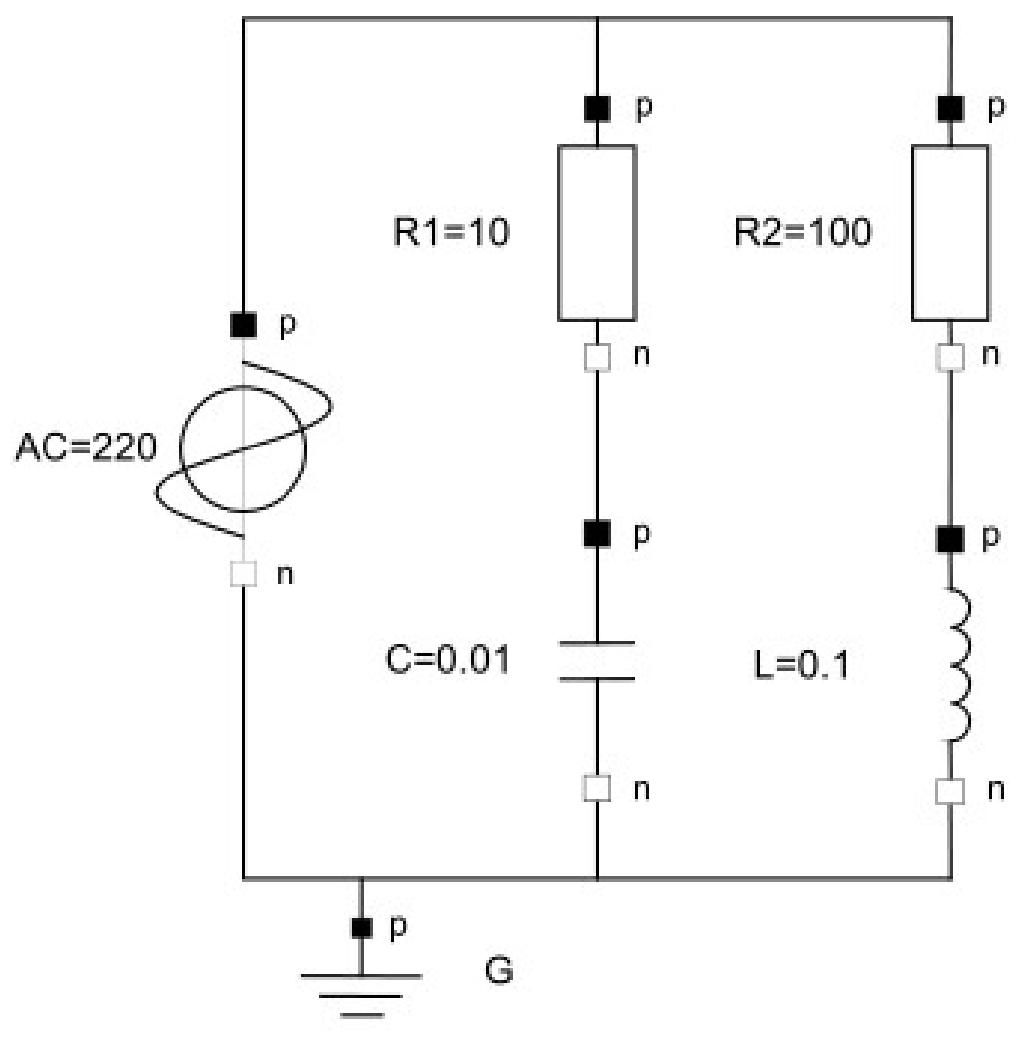

- Modelica: Physical system

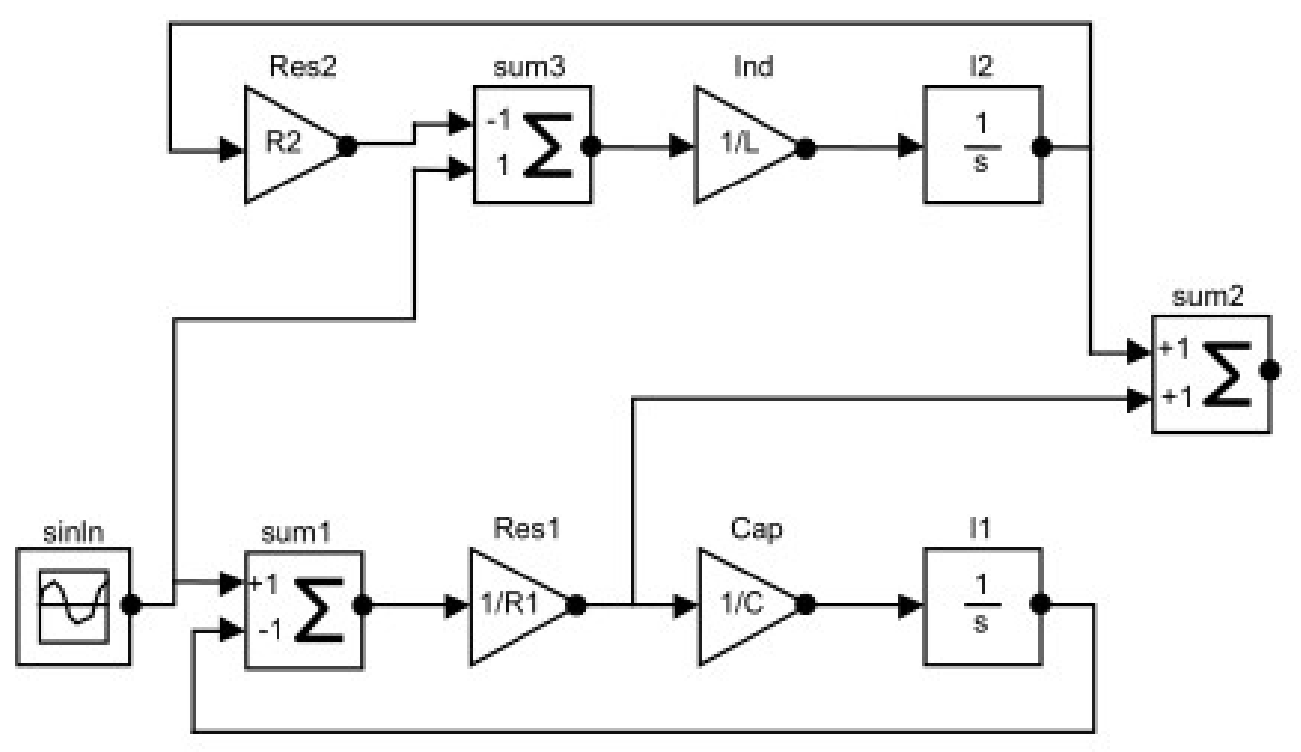

Simulink: Signal-flow model 


\section{Equations versus assignment}

- Equation clauses are not sequences of statements! (in particular, there is no notion of assignment, nor evaluation order)

- It is however possible to describe how to compute a result by means of sequences of assignments, loops, etc. in Modelica, but not using equations! 


\section{semantics}

- The semantics of the Modelica language is specified by means of a set of rules for translating any class described in the Modelica language to a flat Modelica structure. A class must have additional properties in order that its flat Modelica structure can be further transformed into a set of differential, algebraic and discrete equations (= flat hybrid DAE). Such classes are called simulation models. 


\section{Overview}

- Modelica - where to get Information

- Main features

- Building Modelica models

- Some programming details

- Structuring - reuse 


\section{Assembling models}

- To get a model of a system:

- Pick Basic (or complex!) (sub)models

- Connect these models

- The DAE is derivated from the sub-models and from the equations defined by the connections 


\section{Several elementary constructs}

- Model: elementary model or assembly of such models

- Block: construct with defined causality (inputs / outputs)

- Record: data only

- Connector: to define interactions between models

- Function: to define specific computations, behaviors 


\section{A DC motor model}

- A DC motor can be thought of as an electrical circuit which also contains an electromechanical component (Mohsen TorabzadehTari)

model DCMotor

Resistor $\mathrm{R}(\mathrm{R}=100)$;

Inductor $\mathrm{L}(\mathrm{L}=100)$;

VsourceDC DC $(f=10)$;

Ground G ;

ElectroMechanicalElement $\operatorname{EM}(k=10, J=10, b=2)$;

Inertia load;

equation

connect (DC.P,R.n);

connect (R.P, L.n);

connect (L.P, EM.n);

connect (EM.p, DC.n);

connect (DC.n, G.p) ;

connect (EM.flange, load.flange);

end DCMotor

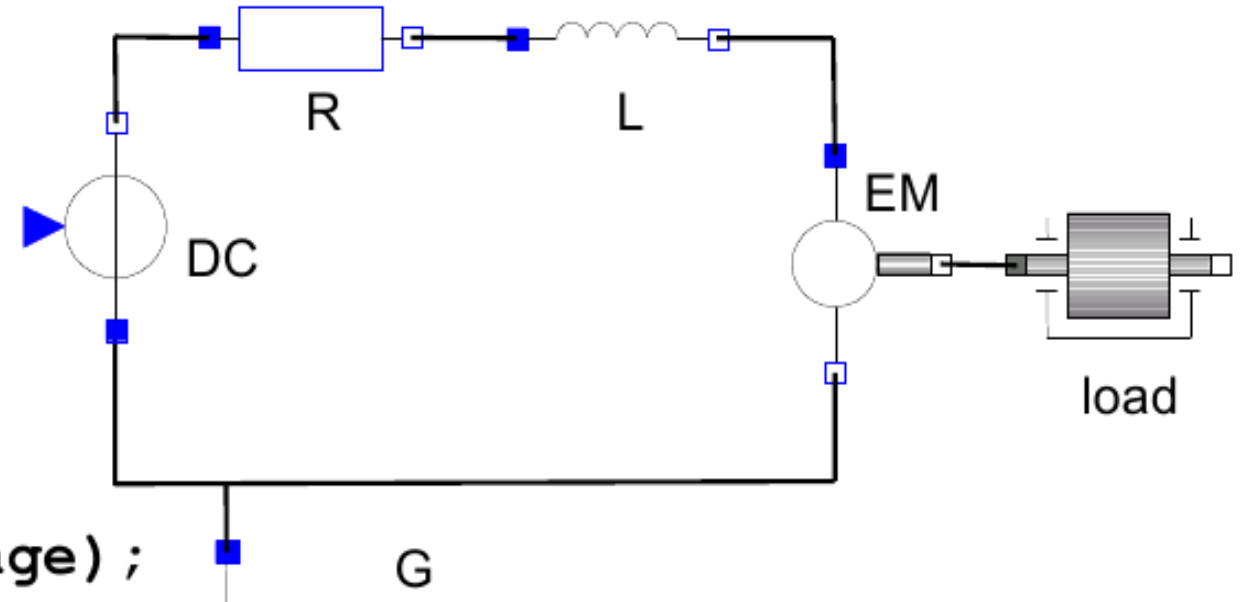




\section{Derived system of equations}

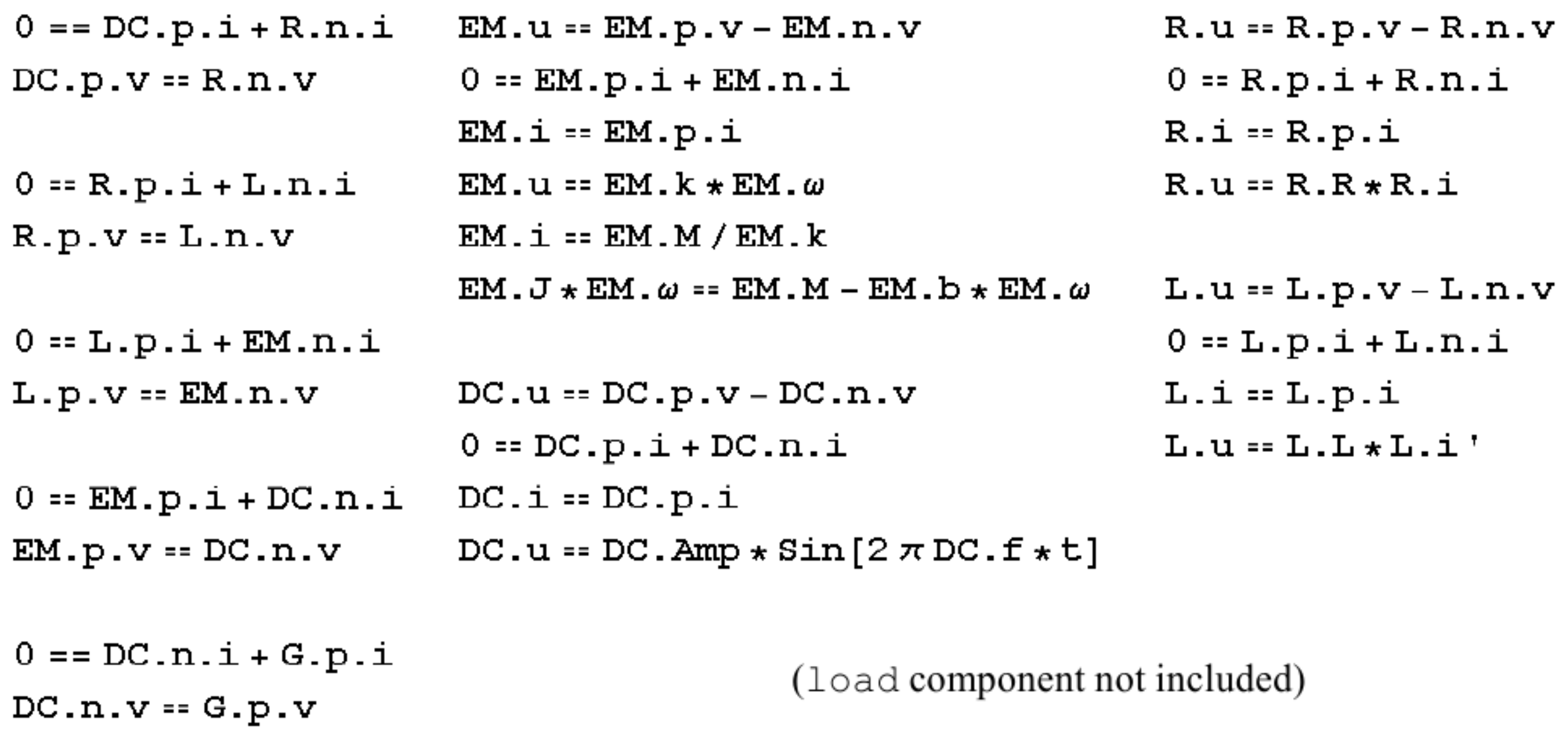

- Automatically derived by a Modelica tool for simulation 


\section{Connectors}

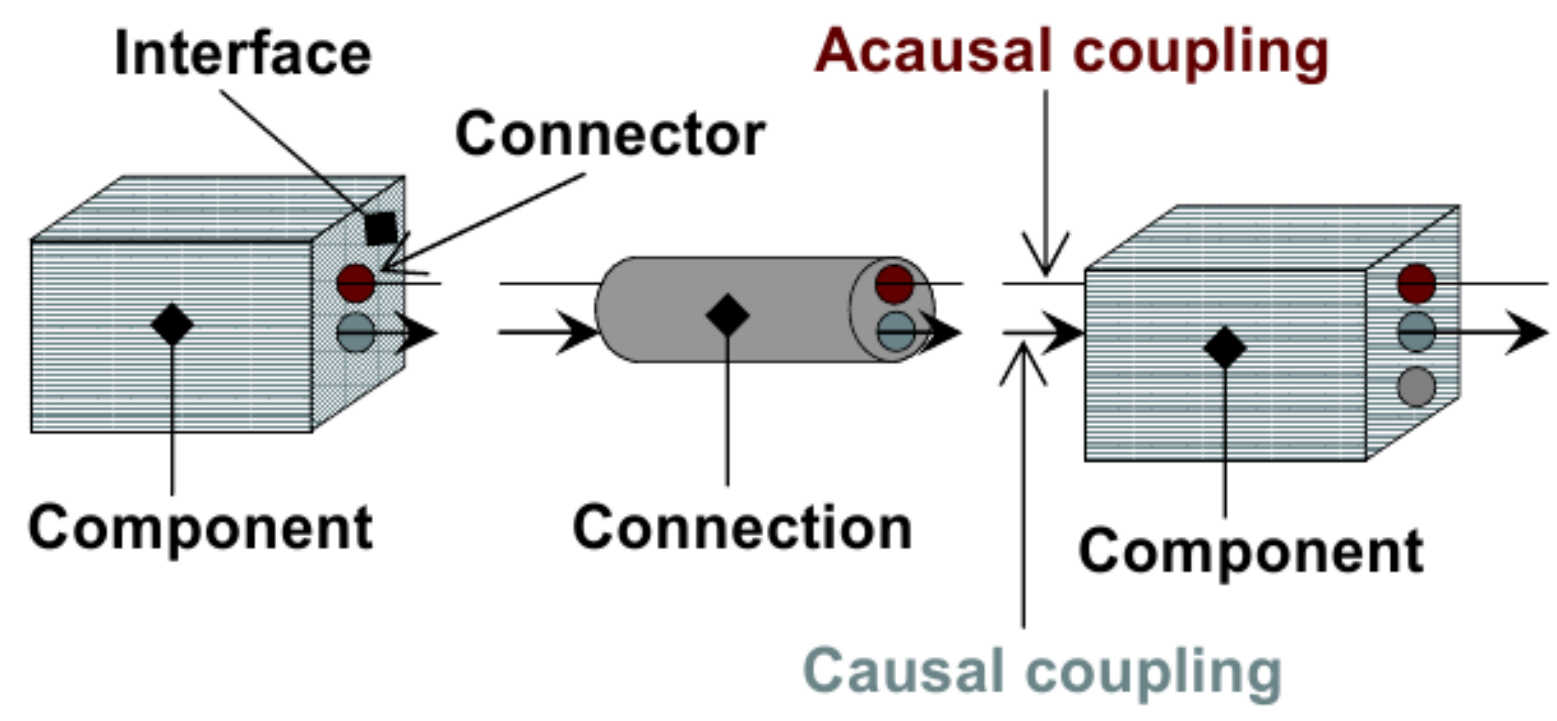

- Connect(A.x, B.y):

- Acausal coupling: $x=y$

- Causal coupling: $x$ and $y$ are declared flow: A.x + B.y $=0$ 


\section{Connector classes}
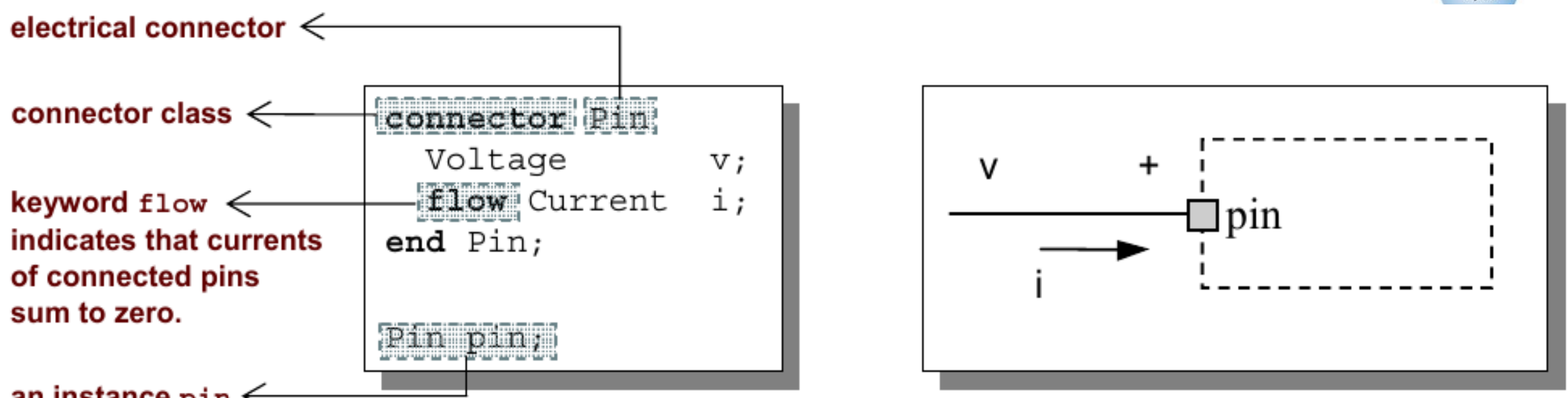

of class Pin

- Connectors are specific classes with two kinds of variables:

- Standard: potential or energy level => Equality

- Flow: some kind of flow => Sum to zero

- Domain Hydraulic Heat

\begin{tabular}{ll} 
Potential & Flow \\
\hline Pressure & $\begin{array}{l}\text { Volume flow } \\
\text { Temp. }\end{array}$ \\
Heat Flow
\end{tabular}

Potential Flow Temp.
Carrier

Volume Heat
Modelica Lib.

HyLibLight

HeatFlowID 


\section{Overview}

- Modelica - where to get Information

- Main features

- Building Modelica models

- Some programming details

- Structuring - reuse 


\section{Algorithms}

- Sequence of statements

- Classical programming constructs:

- Assignments

- If - then - elseif - else

- Loops : for, while

- Event driven statements (see "events"):

- When 


\section{Event based modeling}

- Events are distinguished time instants

- Time instants defined through:

- Explicit time

- Change of variable value : False to True or change of boolean: ( $x>=2.4)$ (edge, change)

- Initial and terminal events

- Repeated events (sample)

- Event based behavior: when cond then equation end when 


\section{Functions (1)}

- Definition of a function:

- Eventually, some structural parameters used to denote array dimensions

- Formal parameters

- Eventually, some internal variables

- Executable statements (or call to foreign function, written in $\mathrm{C}$ for instance)

- Executable statements include assignments, "while" loops, "for" loops and return function myFunction input Real u1, u2; output Real y1, y2; protected Real x1, x2; algorithm

$$
\begin{array}{r}
\mathrm{x} 1:=\mathrm{u} 1+\mathrm{u} 2 ; \\
\mathrm{x} 2:=\mathrm{u} 1-\mathrm{u} 2 ; \\
\mathrm{y} 1:=\mathrm{x} 1 \star \mathrm{x} 2 ; \\
\mathrm{y} 2:=\mathrm{x} 1 / \mathrm{x} 2 ; \\
\text { end myFunction; }
\end{array}
$$




\section{Functions (2)}

- Declarative Mathematical semantics: always returns the same result given the same input argument values

- Positional and named arguments

- Multiple results

- External functions (C or FORTRAN code) 


\section{Overview}

- Modelica - where to get Information

- Main features

- Building Modelica models

- Some programming details

- Structuring - reuse 


\section{Managing complex systems modeling}

- Models: Composition and Hierarchy

- Packages: set of Modelica entities

- Libraries: structured sets of packages

- REUSE! 


\section{Packages}

- A package is

- A name space (model definition, programming)

- A hierarchical set of Modelica classes and constant components

- Packages may be stored:

- As nested Modelica classes, in a single file

- In the host file system, as a tree of directories and files 


\section{Libraries}

- A library is a hierarchy of packages, structured by the application domain or by the provided programming or technical functionalities

- A library usually provides several subpackages containing:

- The public types used in the library

- Eventually, some useful functions

- The connectors used to build classes

- Interfaces of classes

- Instantiable classes

- Some test models 


\section{And now ..}

- Modelica - where to get Information

- Main features

- Building Modelica models

- Some programming details

- Structuring - reuse

- Run the simulation !!! 


\section{Modelica - Modeling and simulation}

Modelica

Graphical Editor

- Global workflow for

modeling and simulation

Modelica

Textual Editor

Cargèse - France - 10-14/04/2012

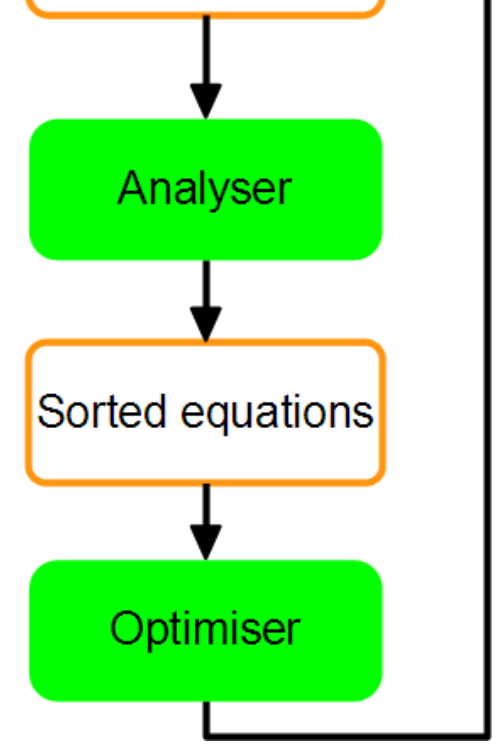

Modelica

Model

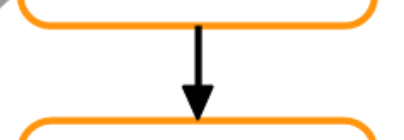

Modelica

Source code

$\downarrow$

Translator

$\downarrow$

Flat model

Hybrid DAE

Optimised

Sorted equations

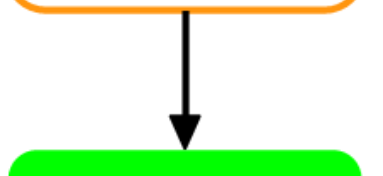

Code generator

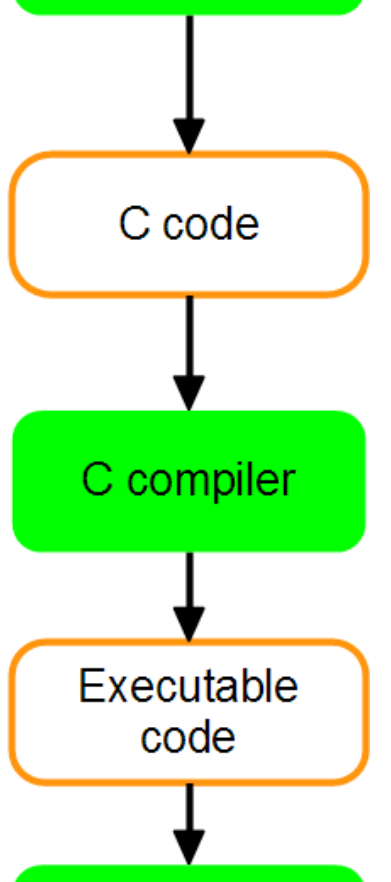

SIMULATION 


\section{Free and commercial Modelica tools}

- Usually provide Modeling AND simulations facilities

- Should provide tool-independent models

- Open source:

- OpenModelica from OSMC

- Commercial

- MathModelica by MathCore

- Dymola by Dassault systems / Dynasim

- SimulationX by ITI

- MapleSim by MapleSoft 


\section{Thank you}

\section{Enjoy modeling/simulation with Modelica!}

\section{Questions?}

